\title{
LONG WAVES IN PARALLEL FLOW IN HELE-SHAW CELLS
}

\author{
Topical Report \\ $\mathrm{DOE} / \mathrm{BC} / 14600--7$ \\ By \\ DE91 002232 \\ Murat Zeybek \\ Yanis C. Yortsos
}

April 1991

Work Performed Under Contract No. DE-FG22-90BC14600

\author{
Prepared for \\ U.S. Department of Energy \\ Assistant Secretary for Fossil Energy
}

Thomas B. Reid, Project Manager

Bartlesville Project Office

P.O. Box 1398

Bartlesville, OK 74005

\author{
Prepared by \\ University of Southern California \\ Department of Petroleum Engineering \\ Los Angeles, CA 90089-1211
}




\section{Contents}

List of Figures $\quad$ iv

1 ABSTRACT 1

2 INTRODUCTION 1

3 FORMULATION 2

3.1 Linear Stability Analysis $\ldots \ldots \ldots \ldots \ldots \ldots$

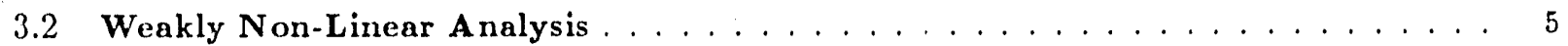

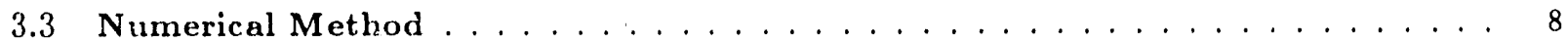

4 EXPERIMENTS AND COMPARISONS WITH THE THEORY

5 CONCLUDING REMARKS 


\section{List of Figures}

1 Flow geometry for (a) non-symmetric and (b) symmetric case . . . . . . . . 16

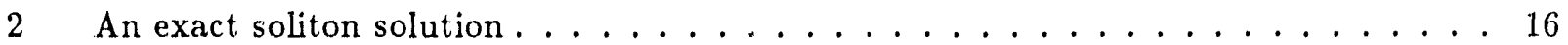

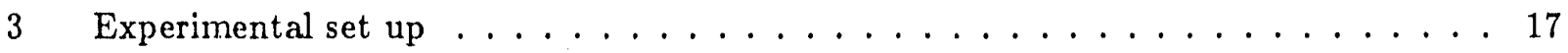

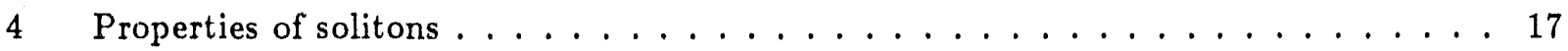

$5 \quad$ Single solitary wave (a) initial condition (b) and (c) subsequent stages $\ldots \ldots \ldots$

6 Numerical simulation of Figure 5 c. Inset: profile in different scales . . . . . . . . 19

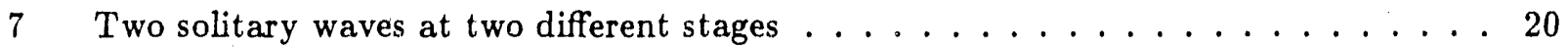

8 Numerical simulation of Figure 7 Inset: Profile in different scales . . . . . . . . 21

9 Two solitary waves (a) before and (b) after interaction . . . . . . . . . . . . 22

10 Numerical simulation of Figure 9. Inset: Profile in different scales . . . . . . . . . 23

11 Short wave initial disturbance and subsequent stage $\ldots \ldots \ldots \ldots \ldots$

12 Numerical simulation of Figure 11. Inset: Profile in different scales . . . . . . . 25

13 Experimental run for $\lambda<1 / 2$ and positive disturbance . . . . . . . . . 26

14 Numerical simulation of Figure $13 \ldots \ldots \ldots \ldots \ldots$

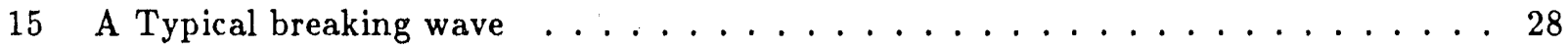

16 A wiggly interface behind the soliton $\ldots \ldots \ldots \ldots \ldots \ldots$ 


\title{
LONG WAVES $\because$ PARALLEL FLOW IN HELE-SHAW CELLS
}

\author{
M. Zeybek and Y.C. Yortsos
}

\section{ABSTRACT}

The evolution of fluid interfaces in parallel flow in Hele-Shaw cells is studied both theoretically and experimentally in the large capillary number limit. It is shown that such interfaces support wave motion, the amplitude of which for long waves is governed by the $\mathrm{KdV}$ equation. Experiments are conducted in a long Hele-Shaw cell that validate the theory in the symmetric case.

\section{INTRODUCTION}

During the past several years the flow of immiscible flow in Hele-Shaw cells and porous media has been investigated extensively. Of particular interest to most studies has been frontal displacement, specifically viscous fingering instabilities and finger growth. This can be readily understood in view of the many interesting theoretical and practical problems associated with such fronts. For Hele-Shaw cells, we mention the selection problems and the singular perturbation associated with the high capillary number limit $[3,6]$, and the relation of viscous fingers to crystal growth [24] and flame front dynamics [34]. In porous media, unstable frontal displacement has been highlighted with the use of Diffusion- Limited-Aggregation (DLA) [28] and other probabilistic growth models [16]. Issues of capillarity, heterogeneity, randomness and spatial correlation, including fractal statistics $[8,18,19,20]$, have been extensively explored, although several outstanding questions still remain unanswered [30!. The practical ramifications regarding oil recovery, as well as many other industrial processes in porous media, have served as the primary driving force for most of these investigations.

By contrast, little attention has been paid to the motion of lateral fluid interface, which are parallel to the main flow direction. Parallel flow is an often encountered, although much overlooked regime. In the context of Hele-Shaw displacement, it is the theoretical limit of fully developed fingers (e.g. the Saffman-Taylor finger [26]) (see Figure 1). Parallel flow conditions have been invoked in qualitative support of the scaling properties of unstable, non-capillary displacement in porous media. Concerning the latter, it has been shown $[15,17]$ that as long as the viscosity ratio $M$ is finite, the initially fractal displacing fluid cluster eventually evolves into a compact Euclidean object (although its perimeter may still be fractal [27]). In a different context, parallel flow is 
often realized in thin and long reservoirs, typically masked under the assumption of vertical flow equilibrium (VFE) $[4,33,29]$. Recent studies on viscous fingering in porous media have made use of parallel flow to interpret numerical experiments $[9,10]$. Finally, we mention that parallel flow is routinely encountered in yet another context, for example in the steady-state, con-current flow processes for relative permeability measurements [5], as well as in processes involving countercurrent imbibition [13]. Although not directly relevant to this work, it must be pointed out that the pore-level analysis of such flows is very much incomplete at present, despite recent efforts [2] to model pore-level viscous coupling between phases and to ascribe a viscosity ratio dependerice on relative permeabilities $[25]$.

It is well known, that under the typical low Re number conditions in Hele-Shaw cells and porous media, single phase (more generally, multi-phase, multi-component flow, but in the absence of spatial and concentration gradients) is potential flow. Such purely viscous flow regimes exist on either side of any interface between immiscible fluids in a Hele-Shaw cell (Figure 1), and sufficiently far from the interface region in the case of porous media. We recall that potential flow (although in the opposite, inviscid limit) also governs the flow in typical water waves [21]. Therefore, the dynamics of the lateral interfaces in parallel flow (such as shown in Figure 1) are likely to be related to those of shallow water waves [12]. The possibility of solitons for Hele-Shaw cells was recently examined in our laboratory. In this chapter we present a preliminary theoretical analysis and related experiments. We should point out that part of the theory was briefly presented in [31], while key experimental findings and comparisons with the theory are discussed in a manuscript submitted for publication [35].

\section{FORMULATION}

We examine the lateral interfaces between two immiscible and incompressible fluids of different viscosities in the parallel Hele-Shaw flow shown schematically in Figure 1. The cell is horizontal and has width $W . \lambda_{1}$ and $\lambda_{2}$ are the normalized interface positions $\left(-1 \leq \lambda_{2} \leq \lambda_{1} \leq 1\right)$. The basic governing equations follow the usual Hele-Shaw assumptions. In each fluid, Darcy's liw applies and the Laplace equation is satisfied for the pressure (velocity potential)

$$
\nabla^{2} p_{i}=0 \quad \Rightarrow \quad \nabla^{2} \phi_{i}=0 \quad i=a, b
$$

Parallel flow requires flat interfaces and the absence of pressure gradients in the transverse $(y)$ 
direction. This condition is satisfied by the requirement $\mu_{b} q_{b}=\mu_{a} q_{a} \equiv Q$. We describe the interfaces by

$$
\mathcal{F}_{i}(x, y, t)=y-F_{i}(x, t)=0 ; \quad i=1,2
$$

On each interface, the following conditions apply: (i) Fluid velocities normal to the interfaces are equal; (ii) Fluid velocities normal to the interface must equal the normal velocity of the interface normal itself. These are expressed as following for each interface

$$
\begin{gathered}
\vec{q}_{b} \cdot \vec{n}_{i}=\vec{q}_{a} \cdot \vec{n}_{i} \\
\vec{q}_{a} \cdot \vec{n}_{i}=-\frac{\mathcal{F}_{i t}}{\left|\nabla \mathcal{F}_{i}\right|} ; \quad i=1,2
\end{gathered}
$$

where $\vec{n}_{i}=\frac{\nabla \mathcal{F}_{i}}{\left|\nabla \mathcal{F}_{i}\right|}$, is the normal vector. Use of Darcy's law transforms the above into the equations

$$
\begin{gathered}
\nabla p_{b} . \nabla \mathcal{F}_{i}=M \nabla p_{a} . \nabla \mathcal{F}_{i} \\
\vec{q}_{a} . \nabla \mathcal{F}_{i}=-\frac{\partial \mathcal{F}_{i}}{\partial t}
\end{gathered}
$$

Across each interface, the pressure drop is due to curvature,

$$
\Delta p_{j}=\gamma \frac{F_{i x x}}{\left|\nabla \mathcal{F}_{i}\right|^{3}} ; \quad j=a, b ; i=1,2
$$

Higher order corrections to pressure drop can be obtained as pointed out by Park and Homsy [23]. However, since the present interest is in long waves, such corrections are of secondary importance.

\subsection{Linear Stability Analysis}

The linearized stability of flat interfaces parallel to the flow direction is next obtained by following an analysis in terms of normal modes. As a preliminary step, we investigate the stability of one interface only, which actually corresponds to the symmetric problem (see Figure $1 \mathrm{~b}, \lambda_{1}=-\lambda_{2}$ and $\eta=-\theta)$. Let $\eta$ and $\theta$ describe the disturbances of the two interfaces. The base state in the absence of disturbances is readily obtained. We have for pressure

$$
\bar{p}_{i}=-q_{i} \frac{\mu_{i}}{k} x \quad ; \quad i=a, b
$$

where $k$ is the permeability of the Hele-Shaw cell, and $\mu_{i}$ is fluid viscosity. The base interface is at location

$$
\bar{F}_{1}=\lambda_{1}
$$


Perturbations are next taken for the pressure and for the interface in terms of normal modes as follows

$$
\begin{gathered}
p_{i}=\bar{p}_{i}+p^{\prime}=-q_{i} \frac{\mu_{i}}{k} x+\varepsilon e^{\omega t+i \alpha x} \varphi_{i}(y) ; \quad i=a, b \\
F_{1}=\bar{F}_{1}+B \varepsilon e^{\omega t+i \alpha x}
\end{gathered}
$$

By a linear analysis and use of Laplace equation with no flow boundary conditions at $y=1$ and $y=0$ the following is obtained

$$
\begin{gathered}
\varphi_{b}=k_{1} \cosh (\alpha(y-1)) ; \lambda_{1}<y<1 \\
\varphi_{a}=k_{2} \cosh \alpha y ; 0<y<\lambda_{1}
\end{gathered}
$$

Substitution of the expressions for perturbed pressure and interface into the interface conditions and subsequent linearization, gives after some algebra the following result for the rate of growth $\omega$ of the perturbation in the $x$ direction

$$
\begin{array}{r}
\omega=-2 i Q \frac{\alpha \sinh \alpha}{\left(\mu_{a}+\mu_{b}\right) \sinh \alpha+\left(\mu_{a}-\mu_{b}\right) \sinh \alpha\left(1-2 \lambda_{1}\right)} \\
-\frac{2 \gamma k \alpha^{3} \sinh \left(\alpha \lambda_{1}\right) \sinh \alpha\left(1-\lambda_{1}\right)}{\left(\mu_{a}+\mu_{b}\right) \sinh \alpha+\left(\mu_{a}-\mu_{b}\right) \sinh \alpha\left(1-2 \lambda_{1}\right)}
\end{array}
$$

Here $M$ is the ratio of the viscosity of fluid $b, \mu_{b}$ to the viscosity of fluid $a, \mu_{a}$. In the above relation, capillarity first enters at $O\left(\alpha^{4}\right)$. For a sufficiently large capillary number, where $N_{c a}=q_{a} \mu_{a} / \gamma$, capillarity can be neglected, although it must be noted that when the capillary number is too large, 3-D effects become important [22] Equivalently, capillarity is negligible for long waves (small $\alpha$ ). At such conditions, the rate of growth is strictly imaginary and the wave dispersion relation at long waves is

$$
\omega \sim-\frac{i \alpha q_{a}}{\left(1-\lambda_{1}+\lambda_{1} M\right)}\left[1+\frac{\alpha^{2}}{3} \cdot \frac{(M-1) \lambda_{1}\left(1-2 \lambda_{1}\right)\left(\lambda_{1}-1\right)}{1-\lambda_{1}+\lambda_{1} M}+\cdots\right]
$$

The above dispersion relation predicts dispersive waves [1] i.e., waves with different wavelength travel with different velocities. This result is different from the previous relations in frontal displacement which yield either growth or decay (viscous fingering). As expected, the waves become non-dispersive, when the fluids have equal viscosity $(M=1)$. Interestingly, non-dispersive waves are also predicted for the Saffman-Taylor conditions $\left(\lambda_{1}=\frac{1}{2}\right)$, although they have infinitesimal velocity when $M \gg 1$. 
An analysis similar to the above can be followed to obtain the dispersion relation for the nonsymmetric case. Two interfaces are considered as shown in Figure 1 a. As expected, a substantially more complicated dispersion relation is obtained. The result is

$$
\tanh \left(\alpha\left(1-\lambda_{1}\right) j\left[-e^{\alpha \lambda_{1}} a \Delta-e^{-\alpha \lambda_{1}} a E\right]-a M e^{\alpha \lambda_{1}} \Delta-b e^{\alpha \lambda_{1}} \Delta+b e^{-\alpha \lambda_{1}} E+a M e^{-\alpha \lambda_{1}} E=0\right.
$$

where

$$
\begin{gathered}
\Delta=e^{-\alpha \lambda_{2}}\left[(b-d) \sinh \left(\alpha\left(1+\lambda_{2}\right)\right)-M d \cosh \left(\alpha\left(1+\lambda_{2}\right)\right)\right] \\
E=e^{\alpha \lambda_{2}}\left[(d-b) \sinh \left(\alpha\left(1+\lambda_{2}\right)\right)-M d \cosh \left(\alpha\left(1+\lambda_{2}\right)\right)\right] \\
a=i \frac{Q}{k}+\frac{\omega}{\alpha} \frac{\mu_{a}}{k} \\
b=i \frac{Q}{k}(1-M) \\
d=i \frac{Q}{k}+\frac{\omega}{\alpha} \frac{\mu_{b}}{k}
\end{gathered}
$$

It is clearly advantageous to consider only the asymptotic expansions at small wave numbers $\alpha$ (large wavelength). We take the expansion $\omega=i Q \alpha\left(x_{0}+x_{1} \alpha+x_{2} \alpha^{2}+\cdots\right)$. As expected, two solutions exist

$$
\omega_{k}=i Q \alpha\left(x_{0, k}+x_{2, k} \alpha^{2}+\cdots\right) \quad ; \quad k=1,2
$$

where $x_{0,1}=-\frac{1}{M}, \quad x_{0,2}=-\frac{2}{\Delta}$

$$
\begin{gathered}
x_{2,1}=\frac{(M-1)\left(\lambda_{1}-1\right)\left(\lambda_{2}+1\right)\left(\lambda_{1}-\lambda_{2}\right)}{M^{2}\left(2+\lambda_{2}-\lambda_{1}\right)} \\
x_{2,2}=\frac{4}{3} \frac{(M-1)}{\Delta^{2}} \frac{\left(\lambda_{1}-\lambda_{2}\right)\left(1+\lambda_{2}-\lambda_{1}\right)\left[\left(\lambda_{2}+1\right)^{2}+\left(\lambda_{1}-1\right)^{2}+\left(\lambda_{2}+1\right)\left(\lambda_{1}-1\right)\right]}{\left(2+\lambda_{2}-\lambda_{1}\right)}
\end{gathered}
$$

and where we have defined $\Delta \equiv 2+(M-1)\left(\lambda_{1}-\lambda_{2}\right)$. In the general case the two roots correspond to the two different amplitude equations for the two interfaces. The results for either symmetric or non-symmetric case, lead to the conclusion that dispersive waves exist at large capillary number values. This interesting finding is subject to further investigation below.

\subsection{Weakly Non-Linear Analysis}

The linear stability analysis is limited to describe the onset of the motion and it is unable to provide further information. The subsequent motion of the waves can be obtained by a non-linear analysis which includes some higher order effects. In this section, the evolution of such dispersive waves is investigated. 
Shallow water waves which describe the motion of waves on the surface of an incompressible fluid bounded by a rigid horizontal plane have been studied in detail [14], [1]. Although the physirs of the Hele-Shaw cell is different from the physics of shallow water waves, similarities can be drawn between the two systems. In particular, we can follow a perturbation analysis valid for small amplitude and long wave length similar to that used for shallow water waves. The initial value problem is solved as explained in [14]. First, dimensionless parameters are defined as follows: $\delta=W / L$, where $L$ is the length of the initial disturbance, $\epsilon=A / W$, where $\mathrm{A}$ is a measure of the disturbance amplitude, $x=X / L, y=Y / W, t=\frac{T}{L / q_{a}}, p=\frac{P}{q_{a} \mu_{a} L / k}, \phi=\frac{\Phi}{q_{a} \mu_{a} L / k}$. (Note that $\delta$ r. as taken to be 1 in the linear stability analysis). In this notation, the Laplace equation in each region satisfies

$$
\delta^{2} \phi_{i x x}+\phi_{i y y}=0, \quad i=a, b
$$

In terms of the dimensionless quantities the interface conditions read

$$
\begin{gathered}
\delta^{2}\left(\phi_{i x}-1\right) \eta_{i x}-\phi_{i y}=\delta^{2} M\left(\phi_{a x}-1\right) \eta_{i x}-M \phi_{a y} \\
\delta^{2}\left(\eta_{i t}-\eta_{i x}\left(\phi_{a x}-1\right)\right)=-\phi_{a y}
\end{gathered}
$$

Equations (17)-(18) apply on the disturbed interface positions, $y_{i}=\lambda_{i}+\eta_{i} \quad i=1,2$. In addition we have:

Continuity of the velocity potential:

$$
\phi_{a}=\phi_{b} \quad \text { at } y_{i}=\lambda_{i}+\eta_{i} \quad ; \quad i=1,2
$$

No flow on the boundaries:

$$
\frac{\partial \phi_{b}}{\partial y}=0 \quad \text { at } \quad y_{i}=\mp 1
$$

Initial condition:

$$
\phi_{b}(x, y, 0)=\phi_{a}(x, y, 0)=0
$$

To implement an asymptotic approach, the long waves approximation is taken, $\delta \ll 1$. Then the following expansion is assumed

$$
\phi_{i}=\phi_{i, 0}+\delta^{2} \phi_{i, 1}+\delta^{4} \phi_{i, 2}+\cdots
$$


Further, assuming small amplitudes $(\epsilon \ll 1)$, the two disturbances $\eta$ and $\theta$ are also expanded

$$
\begin{aligned}
& \eta=\epsilon \eta_{0}+\epsilon^{2} \eta_{1}+\cdots \\
& \theta=\epsilon \theta_{0}+\epsilon^{2} \theta_{1}+\cdots
\end{aligned}
$$

For non-trivial results to be obtained, a relation between $\delta$ and $\epsilon, \delta=\kappa \sqrt{\epsilon}$ is postulated, obtained by the method of dominant balance [14]. The above expansions are subsequently used in the continuity conditions at the interfaces (17)-(18). A large time variable $\tilde{t}=\epsilon t$ is also introduced. After considerable amount of algebra, two coupled systems of PDE's are obtained. The final result is a set of decoupled Korteweg-de-Vries (KdV) and Airy equations

$$
\begin{gathered}
U_{\tilde{t}}-\frac{4(M-1)}{\Delta^{2}}\left(2+\lambda_{2}-\lambda_{1}\right) U U_{\sigma}-\frac{(M-1) \kappa^{2}}{M \Delta} a_{11} U_{\sigma \sigma \sigma}=0 \\
V_{\tilde{t}}-\frac{(M-1) \kappa^{2}}{M \Delta} a_{22} V_{\xi \xi \xi}=0
\end{gathered}
$$

These equations have been decoupled by introducing the following linear transformation of the normalized amplitudes $\eta_{0}$ and $\theta_{0}$, which describe to leading order, interfaces 1 and 2 , respectively (Figure 1)

$$
U=\frac{\eta_{0}-\theta_{0}}{2-\lambda_{1}+\lambda_{2}}, \quad V=-\frac{\left(1+\lambda_{2}\right) \eta_{0}+\left(1-\lambda_{1}\right) \theta_{0}}{2-\lambda_{1}+\lambda_{2}}
$$

However, it should be noted that the equations are not truly decoupled, since the space dependence is expressed in two different moving coordinates $\sigma$ and $\xi$,

$$
\sigma=x-\frac{2 t}{\Delta}, \quad \xi=x-\frac{t}{M}
$$

The various parameters in (22)-(23) are functions of the undisturbed interface positions $\lambda_{1}, \lambda_{2}$, the mobility ratio $M$, and the initial disturbance

$$
\begin{gathered}
a_{11}=\frac{4 M}{3 \Delta} \frac{\left(\lambda_{2}-\lambda_{1}\right)\left(1+\lambda_{2}-\lambda_{1}\right)\left[\left(\lambda_{2}+1\right)^{2}+\left(\lambda_{1}-1\right)^{2}+\left(\lambda_{2}+1\right)\left(\lambda_{1}-1\right)\right]}{\left(2+\lambda_{2}-\lambda_{1}\right)} \\
a_{22}=\frac{\frac{\left(1-\lambda_{1}\right)\left(\lambda_{1}-\lambda_{2}\right)\left(1+\lambda_{2}\right) \Delta}{M\left(2+\lambda_{2}-\lambda_{1}\right)}}{}
\end{gathered}
$$

For the full problem, the solution of both (22)-(23) is required. We may note the following : (i) Purely translational motion occurs when $M=1$, as expected; (ii) In the particular dimensionless 
notation employed, the advective speed $(2 / \Delta, 1 / M)$ decreases to zero as the viscosity ratio increases; (iii) As anticipated, the equations contain the leading orders of the linear analysis in (15); (iv) The dispersive term in the KdV equation vanishes when $1+\lambda_{2}-\lambda_{1}=0$ (which, for the symmetric case $\lambda_{1}=-\lambda_{2}$, coincides with the Saffman-Taylor finger width $\left.\lambda_{1}=1 / 2\right) ;(v)$ Anti-symmetric disturbances $(\eta=\theta)$ are governed by the Airy equation (23) alone; (vi) Finally, when both initial and disturbed interfaces are symmetric $\left(\lambda_{1}=-\lambda_{2}\right.$ and $\left.\eta=-\theta\right)$, the interface evolution is governed by the KdV equation alone. The latter case is of great interest for the subsequent experiments. In terms of the original variables, this particular case reads $\left(\lambda \equiv \lambda_{1}\right)$

$$
\eta_{t}+\frac{2}{\Delta} \eta_{x}-\frac{8(M-1)}{\Delta^{2}} \eta \eta_{x}+\epsilon \frac{4}{3} \frac{\kappa^{2}}{\Delta^{2}}(M-1) \lambda(1-2 \lambda)(1-\lambda) \eta_{x x x}=0
$$

The KdV equation has many interesting properties, in particular the possible development of solitary waves, and has been throughly analyzed by exact techniques of the Inverse Scattering transform ${ }^{11}$.

\subsection{Numerical Method}

Although we shall use general exact results, we shall also rely for illustration purposes on the numerical pseudospectral technique of Fornberg and Whitham [11]. The method combines the Fourier transform treatment of the space dependence with a leapfrog scheme for the time evolution and it is well suited for non-linear dispersive waves. This method assumes that the function $\eta(x, t)$ is periodic in $x$ outside a basic interval $0 \leq x \leq l$. The interval is then discretized by $N$ equidistant points, with spacing $\Delta x=l / N . N$ is taken to be 128 or 256 in the following simulations. The function $\eta(x, t)$ can then be transformed to discrete Fourier space with respect to $\mathrm{x}$ by use of a fast Fourier transform algorithm. Then equation (24) is discretized as follows

$$
\begin{aligned}
\eta_{i}^{n+1}-\eta_{i}^{n-1}=-2 i \Delta & t \frac{2}{\Delta} \mathcal{F}^{-1}\left[\left(\frac{2 \pi}{N} n \mathcal{F}(\eta)\right]+2 i \frac{8(M-1)}{\Delta^{2}}\left(\eta_{i}^{n}\right) \Delta t \mathcal{F}^{-1}\left[\left(\frac{2 \pi}{N} n \mathcal{F}(\eta)\right]\right.\right. \\
& +2 i \epsilon \frac{4}{3} \frac{\kappa^{2}}{\Delta^{2}}(M-1) \lambda(1-2 \lambda)(1-\lambda) \Delta t \mathcal{F}^{-1}\left[\left(\frac{2 \pi}{N}\right)^{3} n^{3} \mathcal{F}(\eta)\right]
\end{aligned}
$$

Since the function is assumed to be periodic, waves that leave the computational domain through one boundary, reenter through the opposite boundary. In the simulations, the computational period is taken to be large enough to avoid any boundary effects. The numerical scheme is tested with an exact soliton solution (Figure 2). It was also tested and found satisfactory with the Zabusky and Kruskal example [32]. 


\section{EXPERIMENTS AND COMPARISONS WITH THE THEORY}

To test the theoretical predictions, a long and narrow Hele-Shaw cell was constructed consisting of two plexiglass plates 0.5 in. thick, of dimensions $220 \mathrm{~cm} \times 27 \mathrm{~cm}$ and of a rubber gasket spacer $0.48 \mathrm{~cm}$ thick and $10 \mathrm{~cm}$ wide. The plates were held together using C-clamps. The experimental set up consists of the Hele-Shaw cell in horizontal position, two integral variable speed peristaltic pumps, a video camera, a video recorder and a monitor (Figure 3). The frame of the cell is constructed such that it can be tilted both with respect to the transverse and longitudinal axes in order to establish a flat interface.

Although other fluid pairs were also used, the experiments reported below were conducted with the pair mineral oil and glycerol/water solution, with corresponding viscosities $170 \mathrm{cp}$ and $860 \mathrm{cp}$, respectively. Oil is the wetting fluid in the experiments. The viscosities of fluids are measured by using both a Cannon-Fenske and a Brookfield spindle viscometer. Since the parallel flow requires $q_{a} \mu_{a}=q_{b} \mu_{b}$, the accuracy of the measurements plays an important role in establishing the flat interface. Relatively high flow rates were used, such that the capillary number was. typirally $O(0.01)$. We must point out that no sustained wave propagation was observed for substantially lower $N_{c a}$ values. This is consistent with the theory. All experiments were conducted with only one interface, as shown in Figures 4-9, hence the conditions pertain to the symmetric case $\left(\lambda_{1}=-\lambda_{2}\right.$ and $\eta=-\theta$ ), the configuration viewed either as the top (or the bottom) half of the symmetric problem. It can be easily checked that the solution of (24) is invariant to the change $\lambda_{1} \rightarrow 1+\lambda_{2}$, $\eta \rightarrow \rho, M \rightarrow 1 / M$, (please note also the rescaling of time), thus without loss we may view the flow as the top half of a symmetric problem with the "lower" fluid being fluid $a$. In our experiments, this was the more viscous fluid, thus $M=0.2$.

Conducting the flow experiments consists of two stages. The first stage is to establish a flat lateral fluid interface and parallel flow conditions for the two fluids. This was accomplished by a displacement process with the aid of gravity. The cell is filled with one fluid first, by tilting the cell and subsequently returning to its horizontal position. While it is being tilted on its side, the lighter fluid is slowly injected at the top. The parameter $\lambda$, which is the dimensionless location of the interface, is controlled by the amount of fluids injected and produced, until the steady interface is established. We stress that the parallel flow condition, $\mu_{b} q_{b}=\mu_{a} q_{a}$, was found essential for the establishment of a parallel interface. When the capillary number is relatively large, the flat 
lateral interface was obtained within $\pm 1 \mathrm{~mm}$ accuracy. In the experiments, integral variable speed peristaltic pumps were used with flow integrators to eliminate pulses.

The second stage is to introduce a disturbance as an initial condition, typically obtained by interrupting momentarily the flow of one fluid, and to monitor the motion of the disturbance. The wave length and the amplitude of the disturbances were controlled by the speed of the interruption. Although results were satisfactory with those initial conditio.s, the questions arise on the effect of flow interruntion and end effects. This will be discussed ir the next section. The existence of solitons can be tested by direct comparison with simulation, but primarily by looking for the following key properties: (i) Arbitrary initial disturhances evolve into one or more solitons and into substantially smaller amplitude dispersive waves; (ii) The speed of a soliton depends on its amplitude, increasing with an increase in amplitude; (iii) Solitons regain their identity after interaction with other solitons (see Figure 4). These were sought in all experiments.

The first experiment to be compared with the simulation is the generation of a single soliton. The zumber of solitons for a given initial condition for a $\mathrm{KdV}$ equation can be predicted by soliton theory [7]. Equation (24) can be transformed into the standard KdV equation

$$
\begin{array}{r}
u_{x}-6 u u_{x}+u_{x x x}=0 \\
u(x, 0)=-u_{0} \operatorname{sech}^{2}\left(b\left(x-x_{0}\right)\right)
\end{array}
$$

The number of solitons is then given by

$$
N \leq\left[\left(\frac{u_{0}}{b^{2}}+\frac{1}{4}\right)^{1 / 2}-\frac{1}{2}\right]+1
$$

A single soliton is predicted with $L=20 \mathrm{~cm}$ (wavelength) and amplitude of $1 \mathrm{~cm}$. Figure 5 shows typical experimental results from still pictures taken from a videotape. A hump-like initial disturbance taken to satisfy the small amplitude and long wave conditions (Figure 5a) is imposed on the parallel interface $(\lambda=0.69)$. Upon restoration of the flow rate to the initial level, the disturbance is first advected by the flow, but also starts developing into a wave of constant shape followed by a wigly interface of small amplitude and short wavelength behind it. For most conditions, this constant amplitude wave has taken a permanent form after traveling about $45 \mathrm{~cm}$, and appears to possess all the characteristics of a soliton (Figure $5 \mathrm{~b}$ ). Its amplitude is clearly different from the initial and remains constant for a substantial distance traveled (Figure 5c), as long as $150 \mathrm{~cm}$, beyond 
which end effects seem to become appreciable. Its velocity to a fixed observer was calculated to be $0.28 \mathrm{~cm} / \mathrm{sec}$, which compares well with the experimental value of $0.26 \mathrm{~cm} / \mathrm{sec}$. This velocity is the sum of that of soliton and the frame of reference velocity, $\frac{2}{\Delta}$. Numerical sinulations corresponding to these conditions and for the initial shape of Figure 5a are shown in Figure 6. One can see that the agreement: between theory and experiment is quite satisfactory, despite the ambiguity on the suitability of the initial condition for the experiment (recall that the disturbance is imposed by flow interruption). The features that are characteristic of soliton and dispersive waves are suppressed in Figure 6, due to the particular scales selected to match one-to-one the experimental pictures. To illustrate this point, the profile of Figure $6 \mathrm{a}$ is replotted in the inset with different scales. We must add that motion of dispersive waves was not observed to our satisfaction in the experiments. Typically, a noisy and wiggly interface of small wave length and amplitude formed soon after the main wave evolved. However, we suspect that wettahility and surface tension played a major role on its subsequent development.

Figure 7 shows the emergence of two solitons arising from an initial disturbance of longer wavelength $(L \sim 45 \mathrm{~cm})$. Accoraing to the theoretical predictions, two solitary waves of different amplitudes (and speed) should arise, with descending order of amplitudes. This sequence is illustrated in Figure 7 which shows the splitting of the initial disturbance, the higher amplitude traveling ahead. As time progresses, the two solitons are clearly separated, the higher amplitude soliton moving faster and away from the trailing lower amplitude soliton. Figure $7 \mathrm{~b}$ shows the lower amplitude soliton. Since the higher amplitude soliton travels faster, it already reaches the end of the cell, when the lower speed soliton is at a distance of $150 \mathrm{~cm}$. Comparison with the numerical simulations is again quite satisfactory (see Figure 8 and the inset with different scales).

Soliton interaction is shown in Figure 9 for the conditions of Figure 6. To create these two solitary waves, two disturbances of different amplitude were sequentially introduced. The second disturbance is of higher initial amplitude and evolves into a faster soliton that eventually takes over the preceding slower one. After this nonlinear interaction, the tall and short solitons reappear, but in reverse order and propagate with their original speed (Figure 9b). All these features are consistent with the theory. Corresponding numerical simulations are shown in Figure 10.

As previously mentioned, a variety of other conditions were also investigated. Short wave disturbances, typically corresponding to $\delta \geq 0.5$ were found to dissipate after their onset, as predicted 
by theory. Figure 11 shows a typical short wave disturbance and a subsequent stage where the arnplitude decreased significantly over the short distance. Figure 12 shows the numerical simulation of Figure 11. The differences between short and long wave disturbances can be readily observed. For $1 / 2<\lambda<1$ and for negative disturbance in the direction opposite to the previous, no solitons are predicted by the theory. This was also tested in the experiments. It was observed that such disturbances (whether long or short wave length) dissipated continuously.

For $0<\lambda<1 / 2$, and negative initial disturbance, solitons propagate in the negative $\sigma$ direction, therefore dispersive waves should proceed them in a fixed frame of reference. Although such effects were noticed and the velocity agreed with the theory to some degree, solitons were not observed. Part of the reasons for the discrepancy must be the method for introducing the disturbance (very near the injection port). Another reason may be wettability. Preliminary experiments with different pair of fluids in which the solitons propagate in the negative $\sigma$ direction (equivalent to this case), do not show the same behavior. This will hopefully be clarified by running experiments with fluid pairs of identical viscosities, but with opposite wettabilities. On the other hand, for $0<\lambda<1 / 2$ and positive initial disturbance, no solitons are predicted by the theory. Figure 13 shows one experimental run, Figure 14 containing the simulation results. It is observed that the decrease in amplitude is slower than in the case of short waves.

We might add that finite amplitude equations describing breaking waves were also derived. Such waves have been frequently observed in our experiments, but will not be discussed further in this report. It has been observed that large amplitude waves, typically with $\epsilon>0.35$, break in all runs (Figure 15). Although not conclusive, our preliminary results support the theory, This subject will be discussed in a future study.

For $M=1$, both the non-linear and the dispersive terms in the $\mathrm{KdV}$ equation (24) are zero, hence we expect constant wave speed and translational motion independent of the wave amplitude. Experiments were run for nearly equal viscosity fluids. We introduced the same disturbance as shown in Figure 5. Now, however neither a change in shape nor a wiggly inierface were observod. As a second test, two disturbances with different amplitudes were also sequentially introduced, in a way similar to the soliton interaction case. It was observed that the distance between the two disturbances remained unchanged. The two disturbances propagated with the same velocity, which also agreed with the theoretical value $\frac{2}{\Delta}$. Furthermore, under this condition, large amplitude waves 
did not break, in contrast to every other case invectigated. This is also consistent with the theory.

\section{CONCLUDING REMARKS}

As mentioned above, initial disturbances were introduced by flow interruption in all runs. At this point, this effect which violates the condition of parallel flow is not clear. To check the effect of the initial disturbance, the data of $4 \mathrm{~b}$ is used as an initial condition in the simulations. The result obtained compared very well with Figure 4c. Although this may suggest that such effect are not significant, future experiments will be carried out with disturbances introduced far from the ends.

It was pointed out that dispersive waves are associated with solitons as a result of the arbitrary initial condition. These waves $c$ in be identified in the simulations, but only if they are plotted in larger scales. Due to their small amplitudes, they are suppressed in the scales that match experiments. Such waves are not observed in the experiments. However, a wiggly shape did develop at the points where it was supposed to be present (Figure 16). This was observed in both forward and backward moving solitons. We believe that the motion of dispersive waves initiates such interface. However, the subsequent motion is largely controlled by surface tension effects which become important at small wavelengths. No wiggly interface was noted in the experiments for equal viscosity fluids $(M=1)$. The observation of such dispersive waves can be rather cumbersome due to surface tension effects. It shculd be recalled that even in shallow water waves they are detected only by special probes.

The results reported above explored a small, although important, subset of parallel flow in Hele-Shaw cell. It is important to point out that this is the first time that Hele-Shaw (and, perhaps, porous media) flnws have been reported to contain KdV dynamics, in addition to their other interesting properties cited in the Introduction. Full validation of the theory can be achieved by extenting the current set up to the non-symmetric case. This can be accomplished by two fluids (relatively easier) and by three or more fluids, if possible. The experiments with two fluids/two interfaces are underway. Such will be an interesting experiment not only from an experimental but also from a theoretical point of view. Of course, our ultimate oljective involves extension of the previous findings to porous media. 


\section{References}

[1] M.J. Ablowitz and H. Segur. Solitons and the Inverse Scattering Transforms. SIAM, 1981.

[2] R.W. Aul and W.L. Olbright. J. Fluid Mech., 215(585), 1990.

[3] D. Bensimon, L.P. Kadanoff, S. Liang, B.I. Shraiman, and C. Tang. Rev. Mod. Phys., 58(977), 1986.

[4] K.H. Coat, J.R. Dempsey, and J.H. Anderson. SPEJ, (63), 1971.

[5] R.E. Collins. Flow of Fluids Through Porous Media. Petroleum Publishing Co. Tulsa, OK, 1976.

[6] R. Combescot, T. Dombre, V. Hakim, Y. Pomeau, and A. Pumir. Phys. Rev. Let., 56(2036), 1986.

[7] P.G. Drazin and R.J. Johnson. Solitons: An Introduction. Cambridge University Press, 1989.

[8] A.S. Emanuel, G.K. Alameda, R.A. Behrens, and T.A. Hewett. SPERE, 4(311), 1989.

[9] F.J. Fayers. SPERE, (551), 1988.

[10] F.J. Fayers and T.M.J. Newley. SPERE, (542), 1988.

[11] B. Fornberg and G.B. Whitham. Proc R. Soc.Lond., 289, 1978.

[12] J. Hammack, N. Scheffner, and H. Segur. J. Fluid Mech., 209(567), 1989.

[13] F. Kalaydjian and B. Legait. C.R.A.S., 304(869), 1987.

[14] J. Kevorkian and J.D. Cole. Perturbation Methods in Applied Mathematics. Springler-Verlag, 1980.

[15] M.J. King and H. Sher. Phys. Rev., A 41(874), 1990.

[16] P.R. King. J. Phys, 20(1529), 1987.

[17] J. Lee, A. Coniglio, and H.E. Stanley. Phys. Rev., A 41(4589), 1990.

[18] R. Lenormand. Proc. Roy. Soc., A 23(159), 1989. 
[19] R. Lenormand, F. Kalaydjian, M.T. Bieber, and J.M. Lombard. paper SPE 20475 presented at the 66th SPE Annual Conference, New Orleans, LA, Sept. 22-26, 1990.

[20] R. Lenormand,-E. Touboul, and C. Zarcone. J. Fluid Mech., 189(165), 1988.

[21] J. Lighthill. Waves in Fluids. Cambridge University Press, 1978.

[22] T. Maxworthy. Phys. Rev. A, 39(5863), 1989.

[23] C.W. Park and G.M. Homsy. J. Fluid Mech: 139(291), 1984.

[24] P. Pelcé. Dynamics of Curved Fronts. Academic Press, 1988.

[25] D.H. Rothman. J. Geophys. Res., 59(8663), 1990.

[26] P.G. Saffman and G.I. Taylor. Proc. Roy. Soc. London Ser., A 245(312), 1958.

[27] H. Siddiqui and M. Sahimi. Chem. Eng. Sci., 45(163), 1990.

[28] T.A. Witten and L.M. Sanders. Phys. Rev. Let., 47(1400), 1981.

[29] Y. Yokoyama and L.W. Lake. paper SPE 10109 presented at the 56th Annual SPE Conference, San Antonio, TX, Oct. 5-7, 1981.

[30] Y.C. Yortsos. to appear. J. Phys.: Condens. Matter, 2(SA 443-S A 448), 1990.

[31] Y.C. Yortsos and M. Zeybek. paper presented at the 1989 AIChE Fall Meeting, San Francisco, CA, Nov. 5-10, 1989.

[32] N.J. Zabusky and M.D. Kruskal. Physical Review Letters, 15(6), 1965.

[33] V.J. Zapata and L.W. Lake. paper SPE 10111 presented at the 56th Annual SPE Conference, San Antonio, TX, Oct. 5-7, 1981.

[34] Y.B. Zeldovitch. Comb. Sci. and Tech., (241), 1980.

[35] M. Zeybek and Y.C. Yortsos. submitted. Phys. Rev. Lett., 1991. 


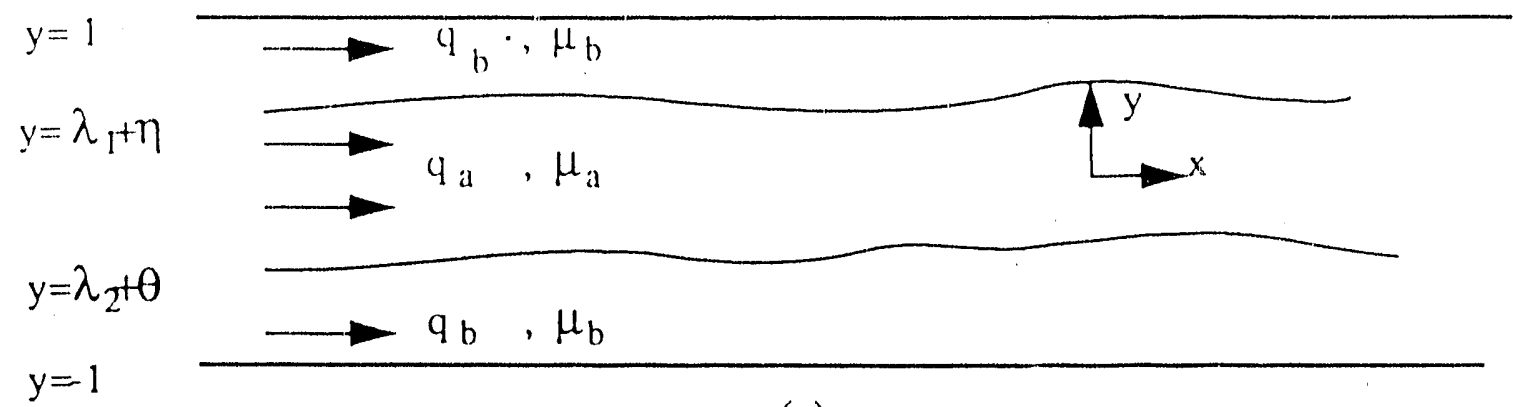

(a)

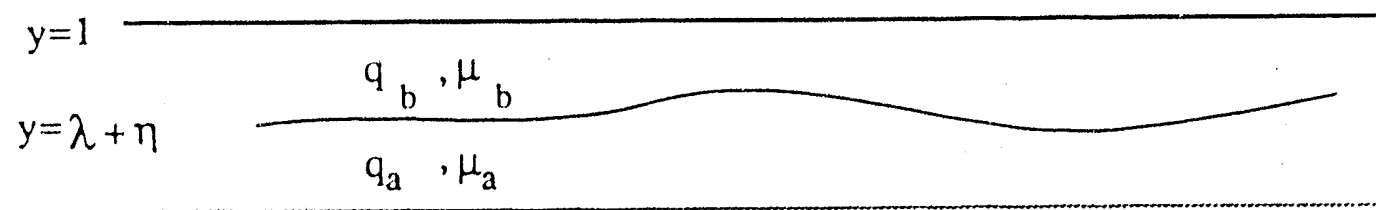

$y=0$

(b)

Figure 1: Flow geometry for (a) non-symmetric and (b) symmetric case

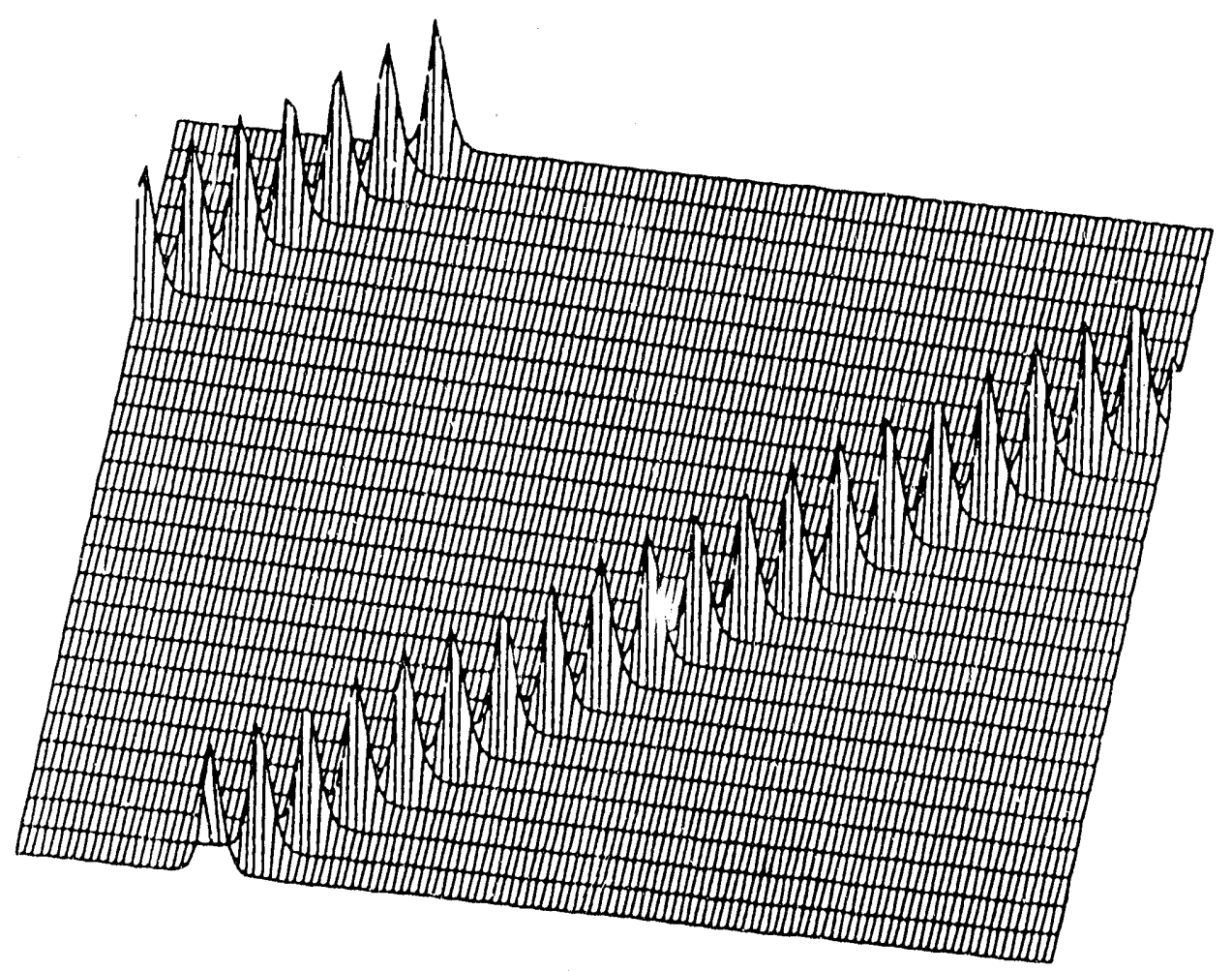

Figure 2: An exact soliton solution 

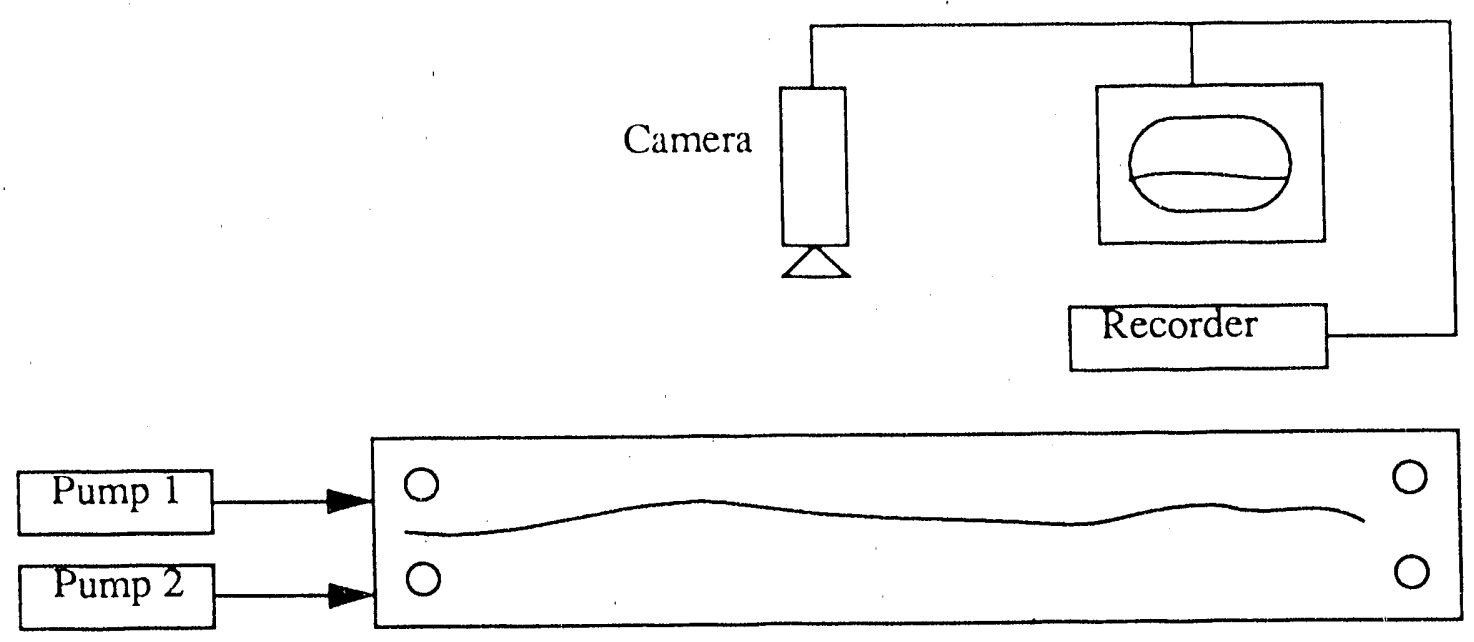

Figure 3: Experimental set up
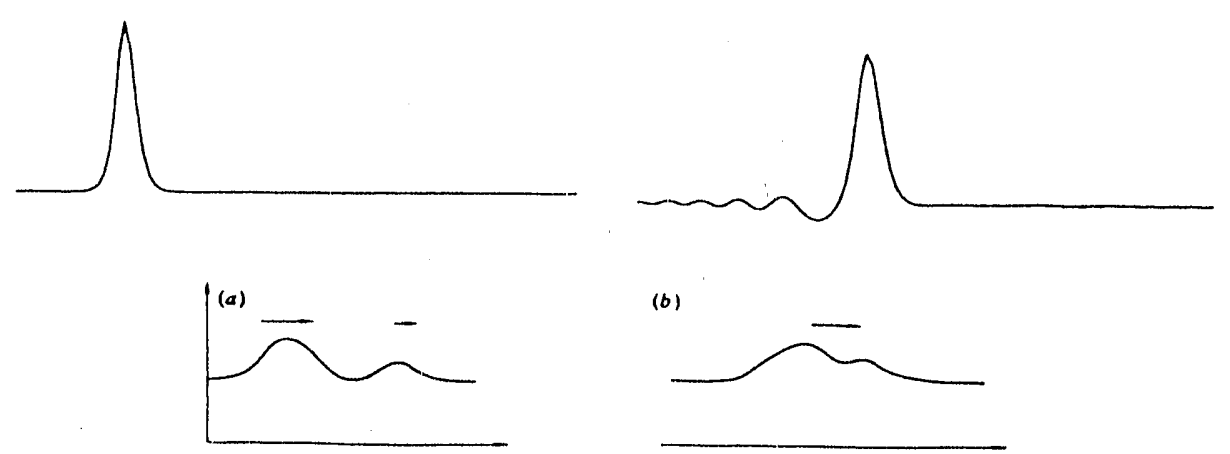

(b)
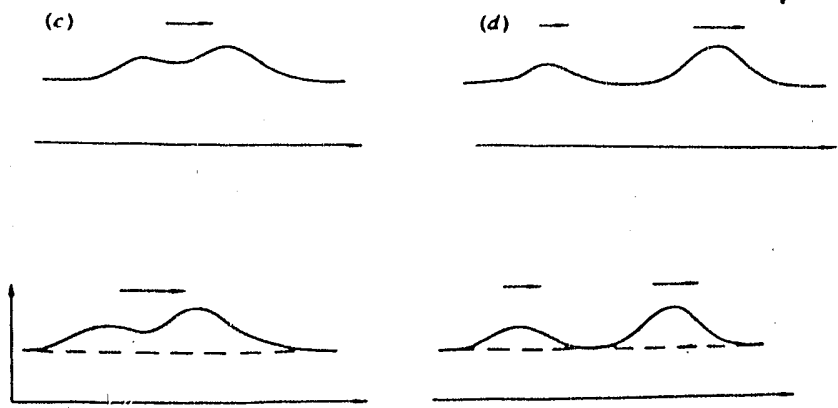

Figure 4: Properties of solitons 

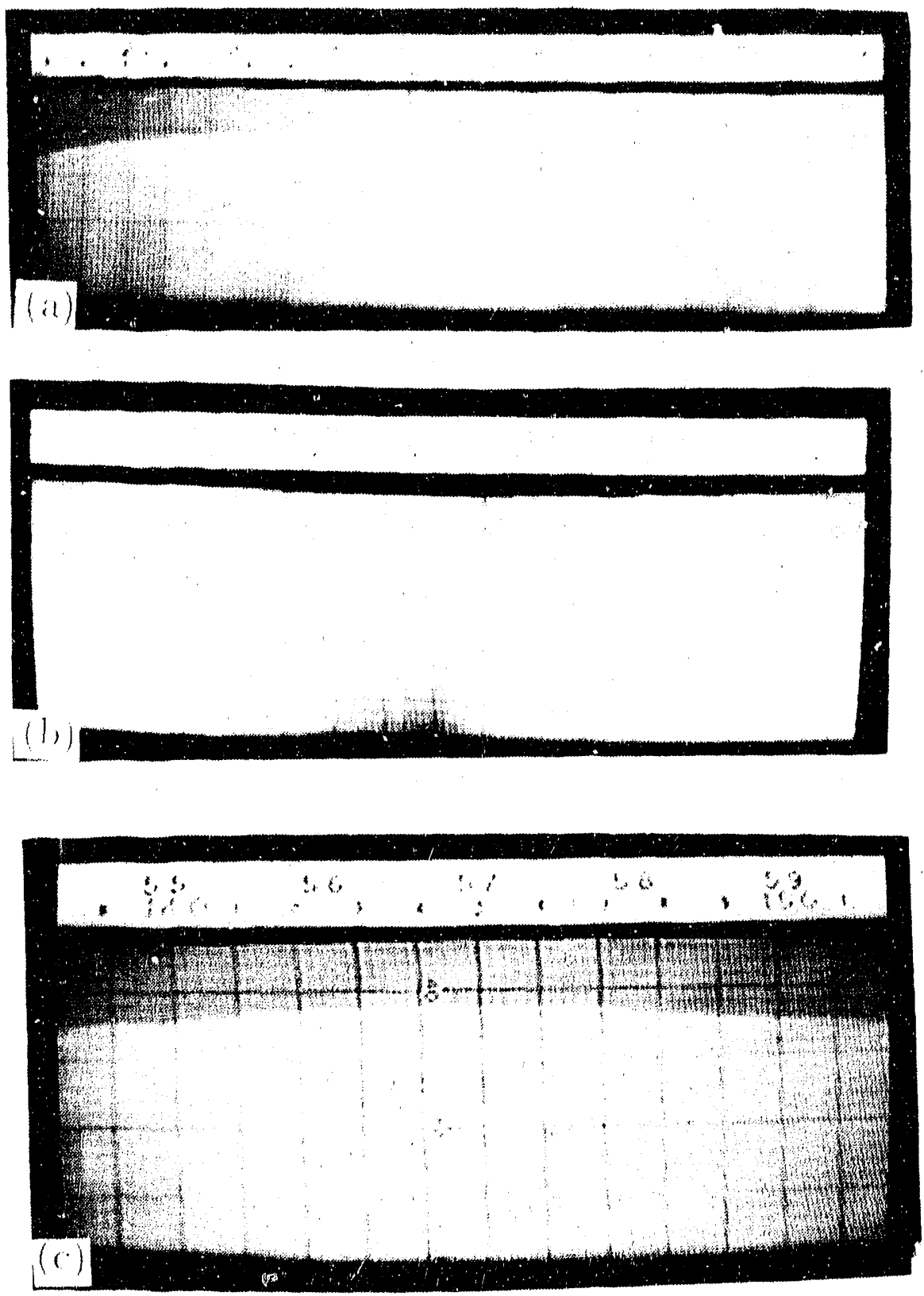

Figure 5: Single solitary wave (a) Initial condition (b) and (c) subsequent stages 


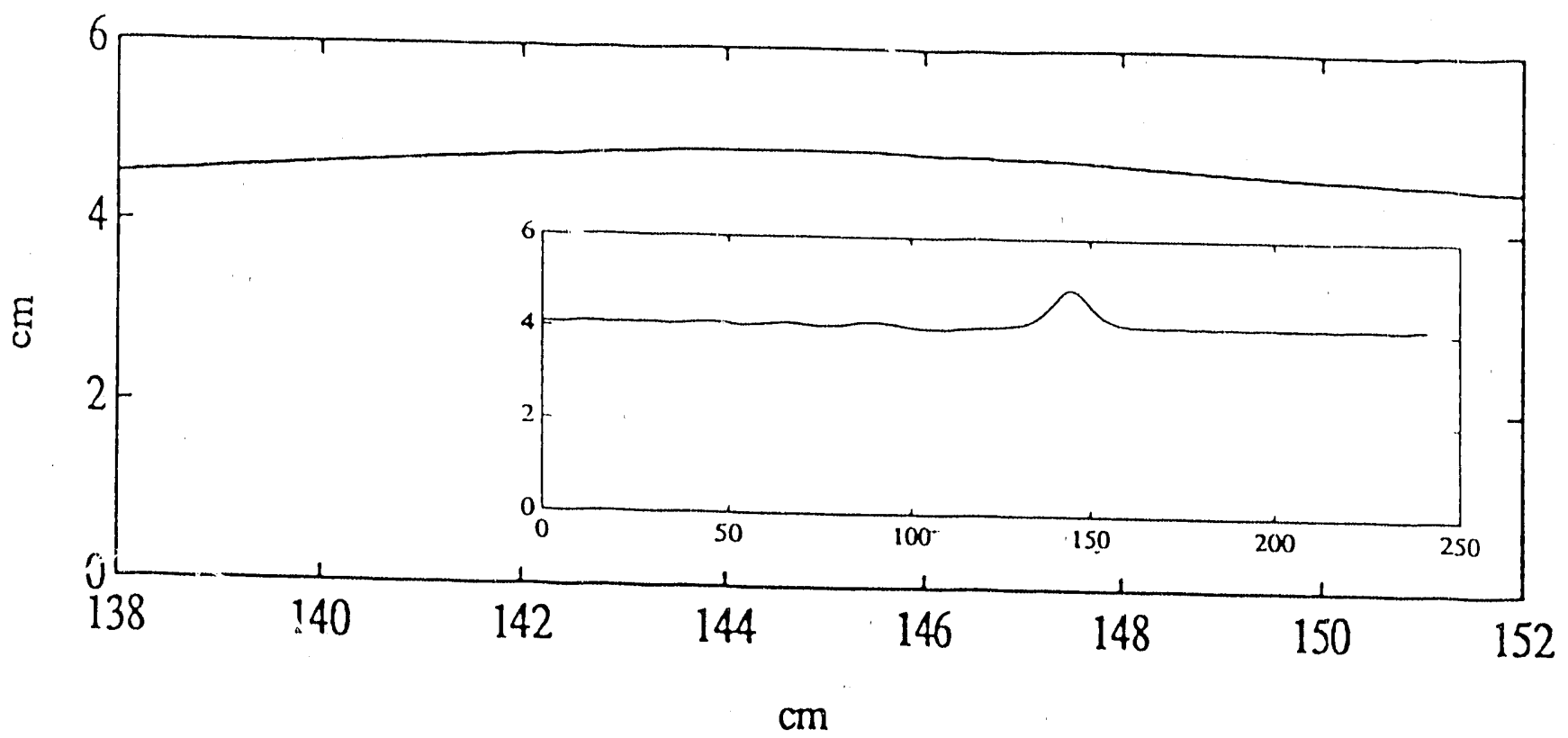

Figure 6: Numerical simulation of Figure 5 c. Inset: profile in different scales 

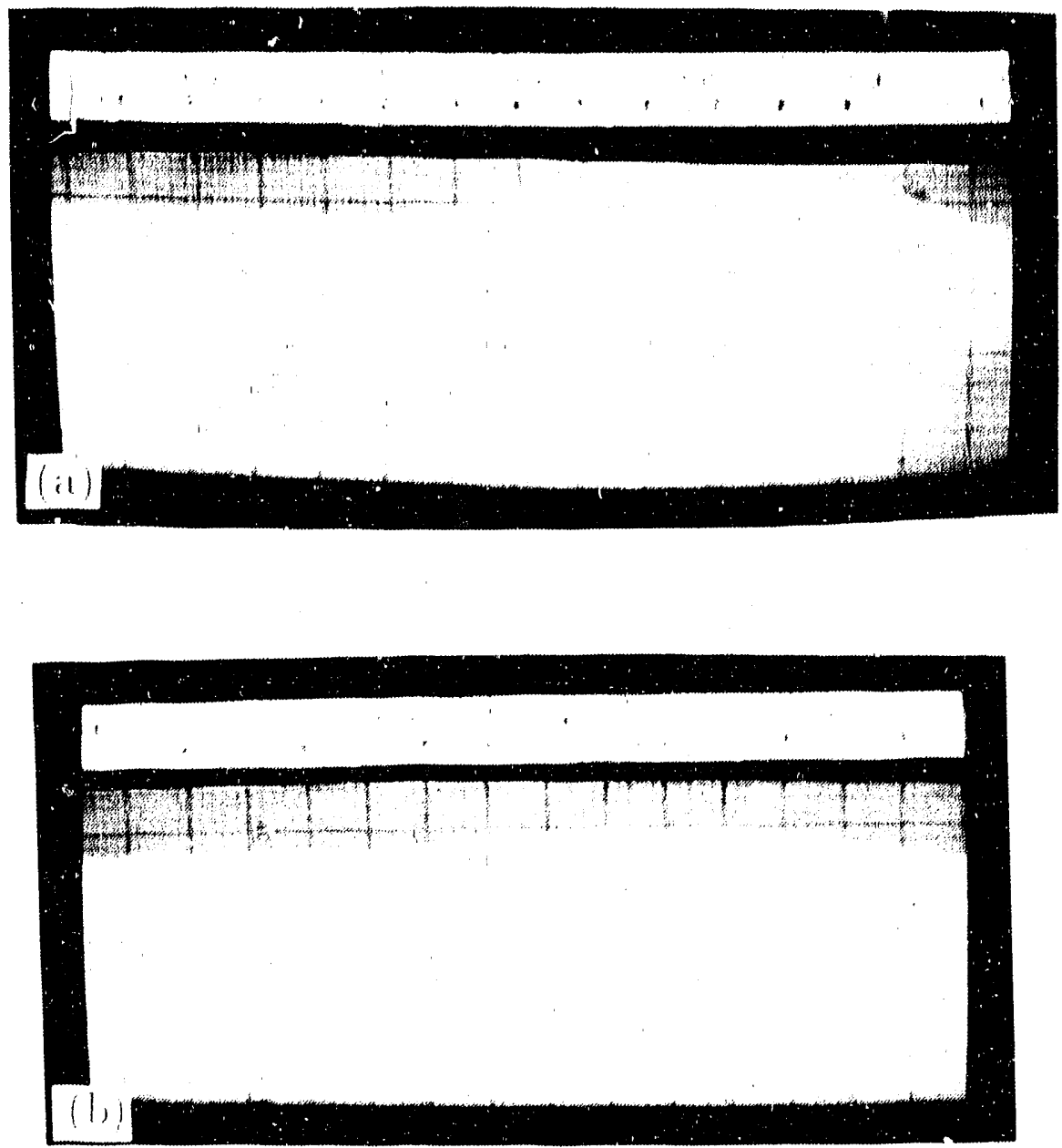

Figure 7: Two solitary waves at two different stages 

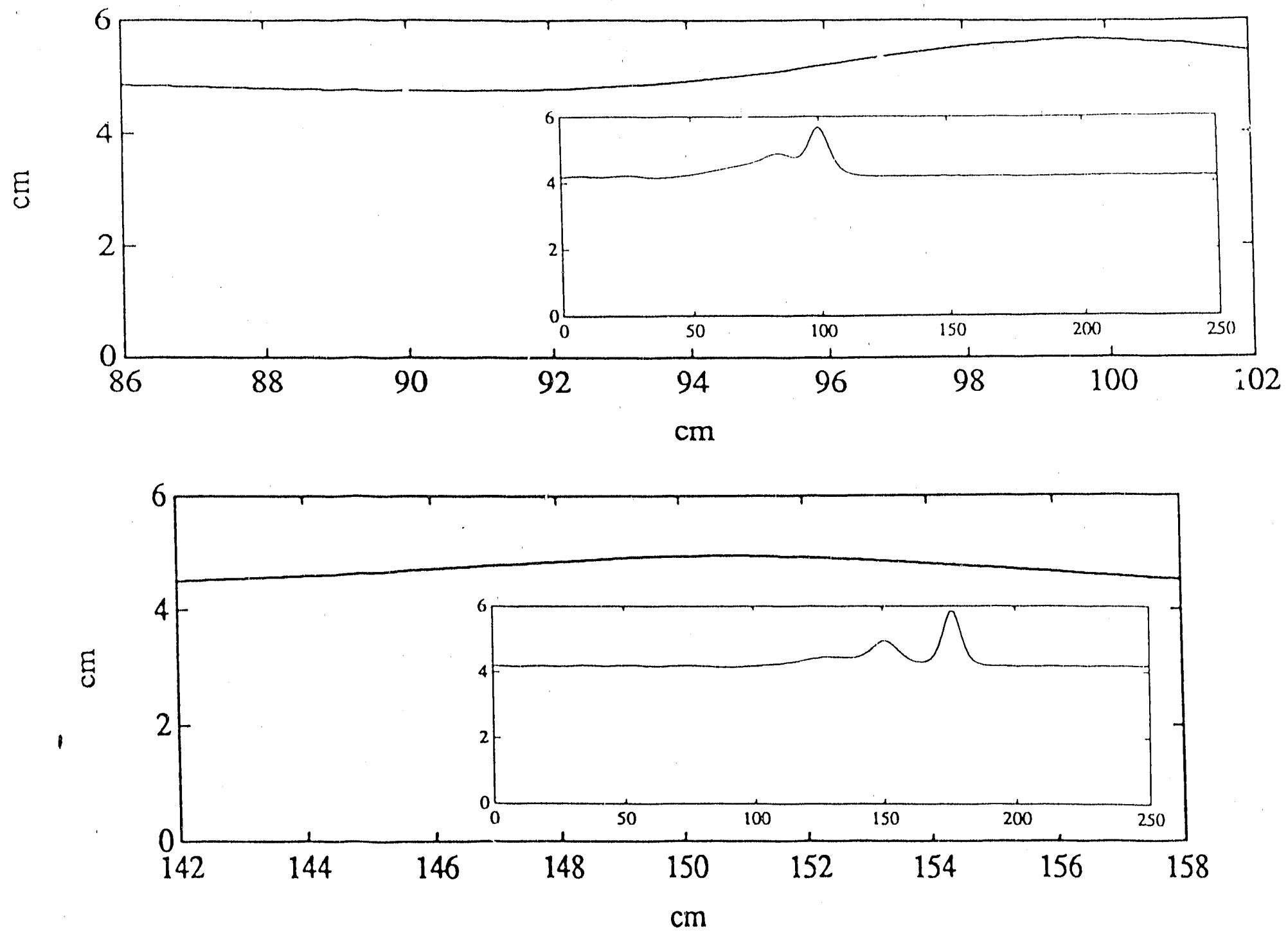

Figure 8: Numerical simulation of Figure 7 Inset: Profile in different scales 

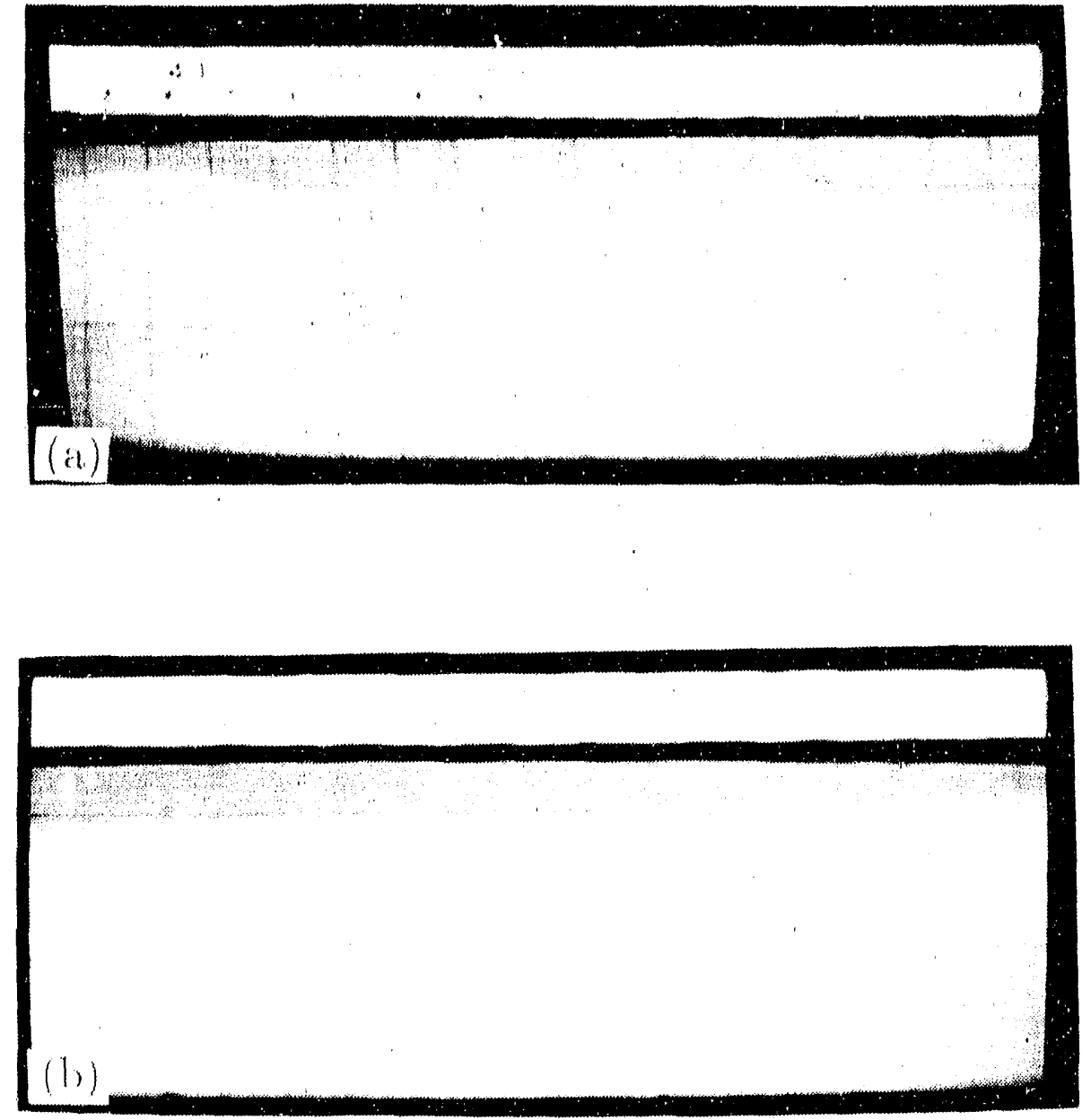

Figure 9: Two solitary waves (a) before and (b) after interaction 

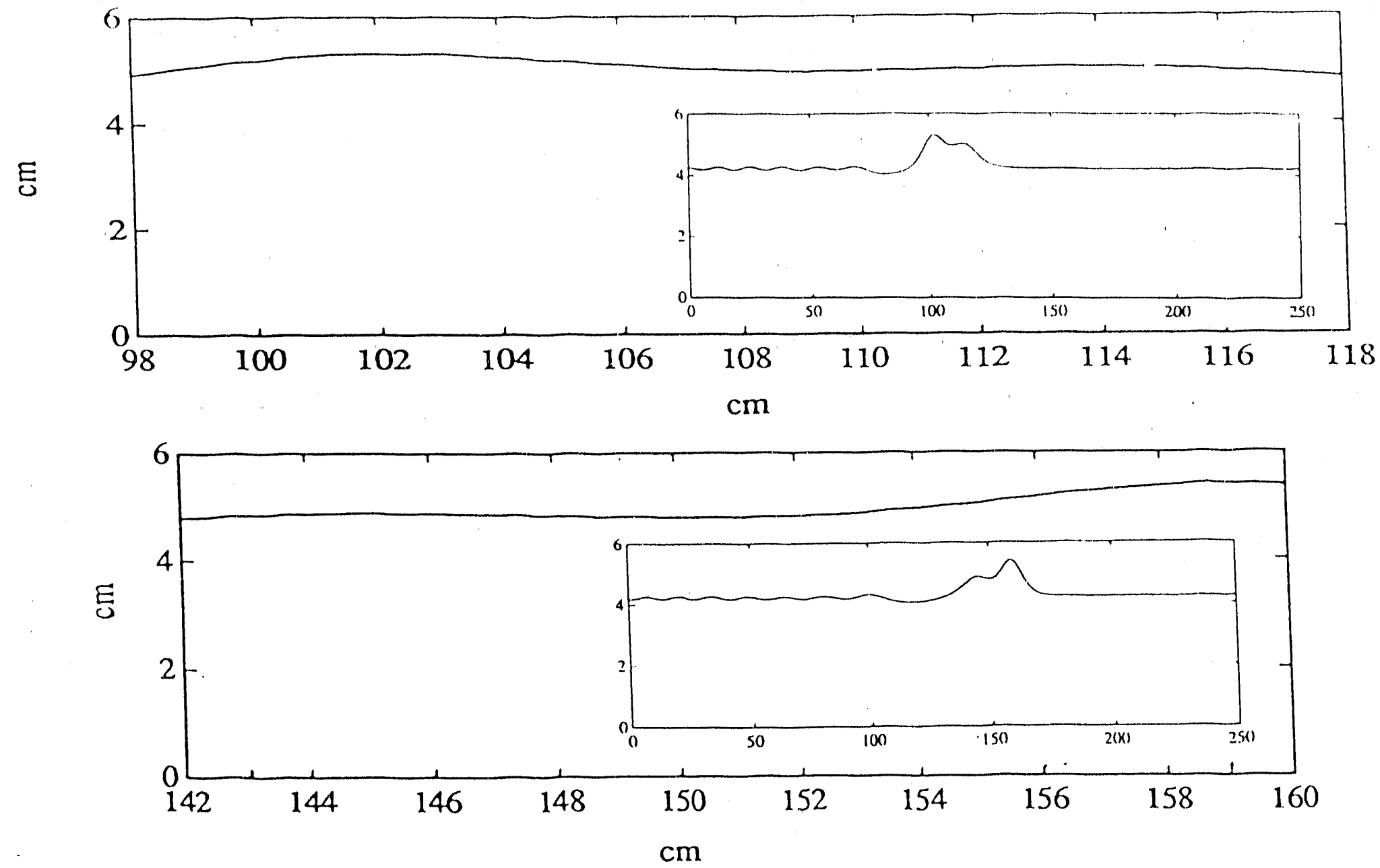

Figure 10: Numerical simulation of Figure 9. Inset: Profile in different scales 

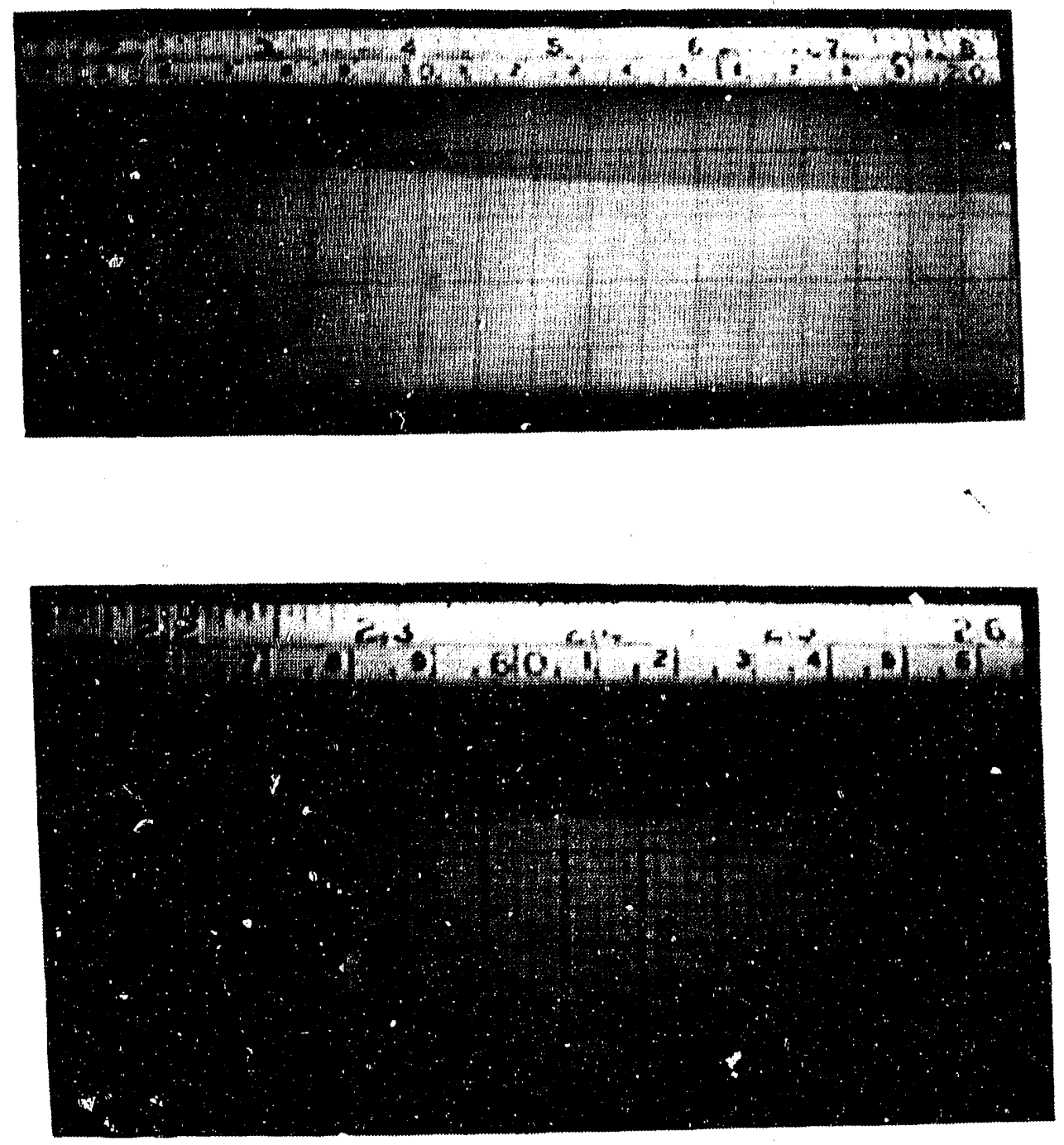

Figure 11: Short wave initial disturbance and subsequent stage 

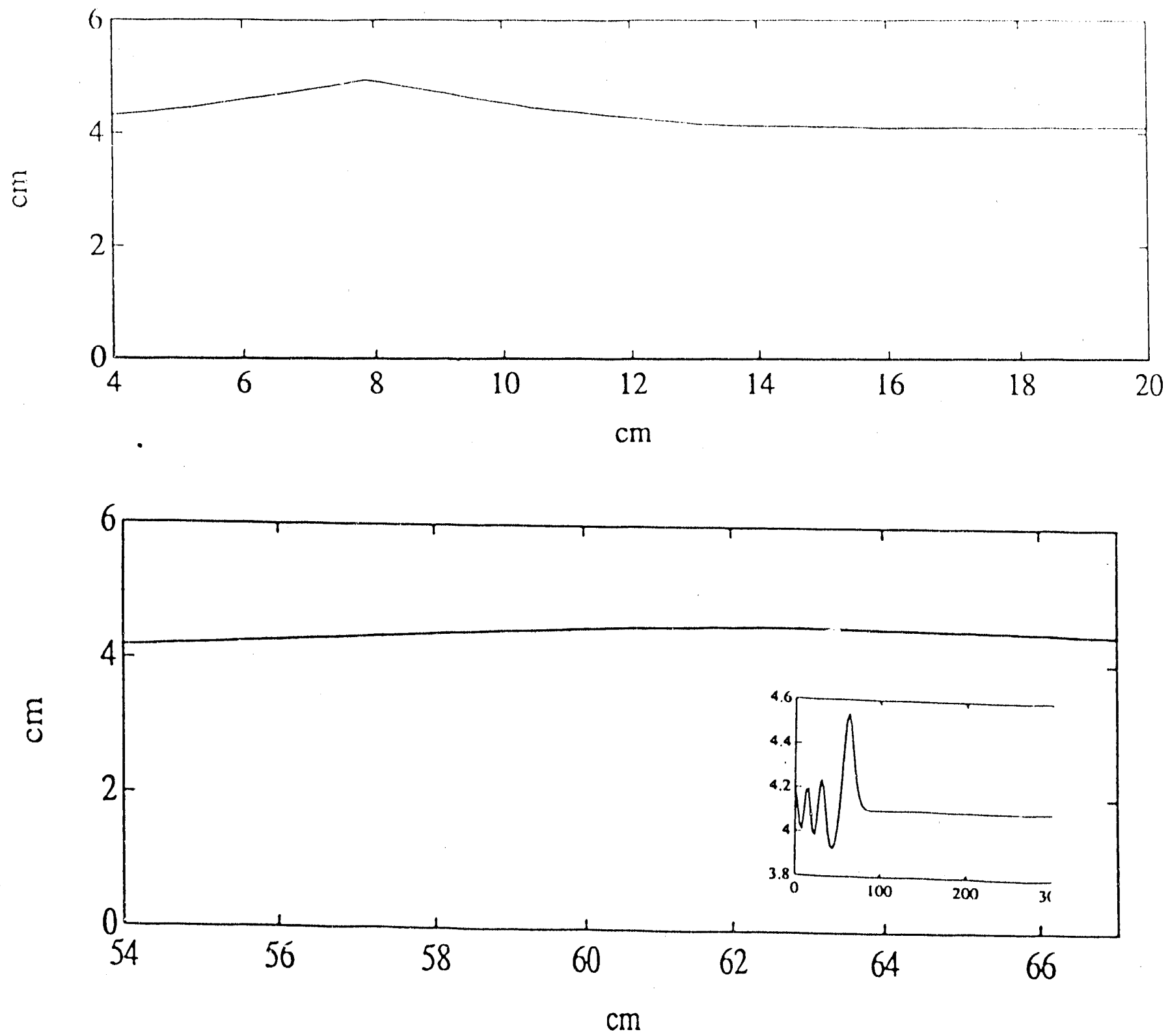

Figure 12: Numerical simulation of Figure 11. Inset: Profile in different scales 

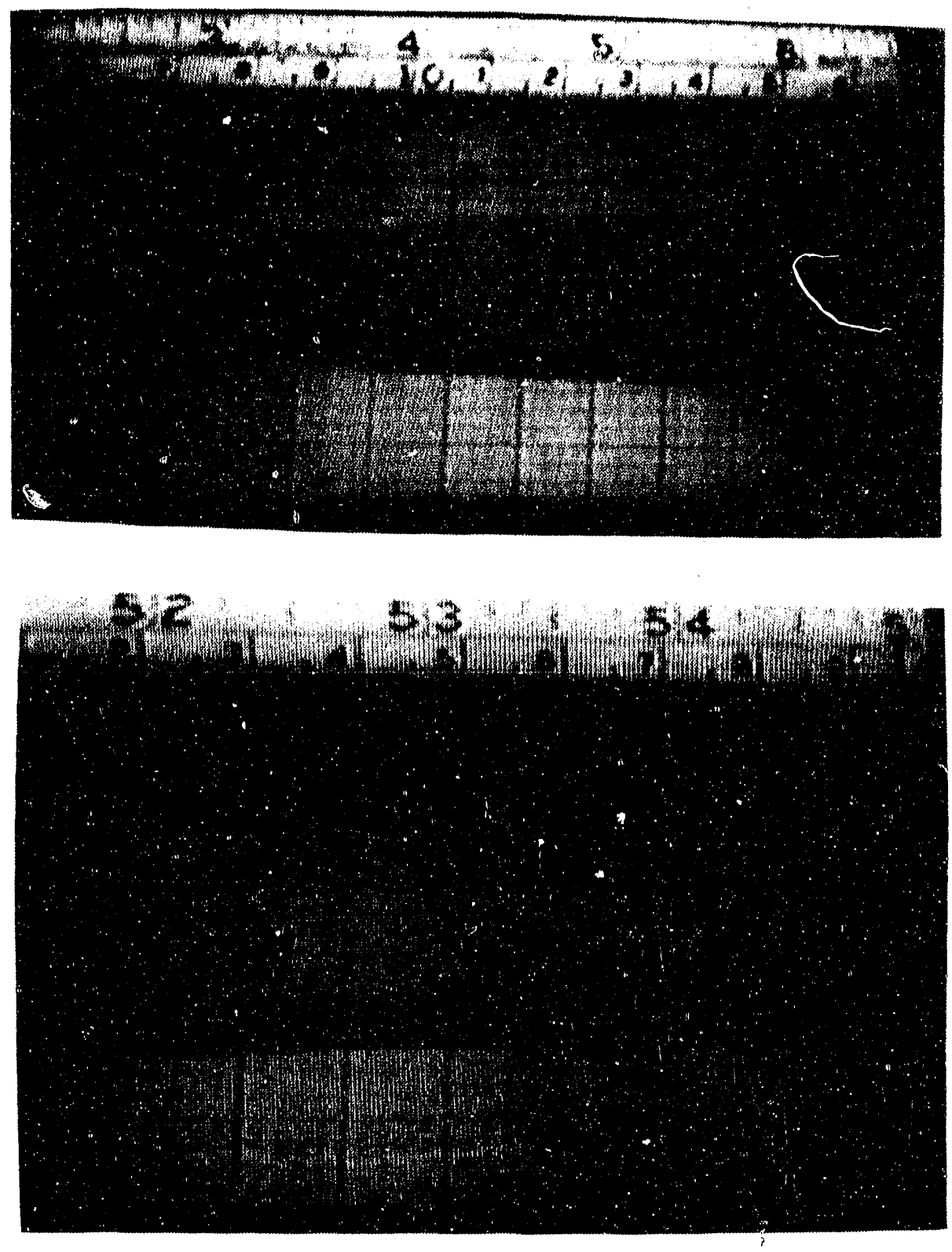

Figure 13: Experimental run for $\lambda<1 / 2$ and positive disturbance 

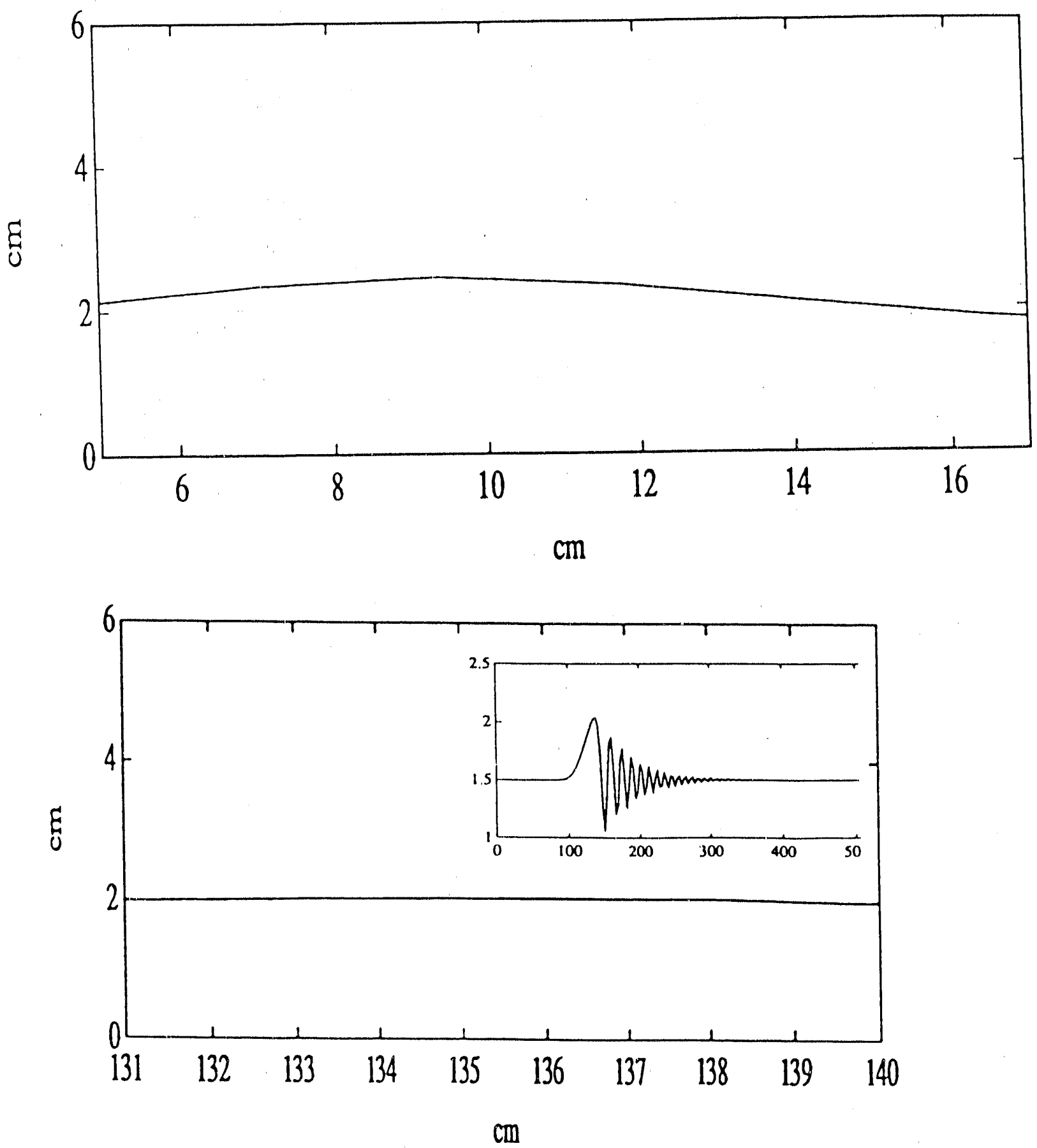

Figure 14: Numerical simulation of Figure 13 


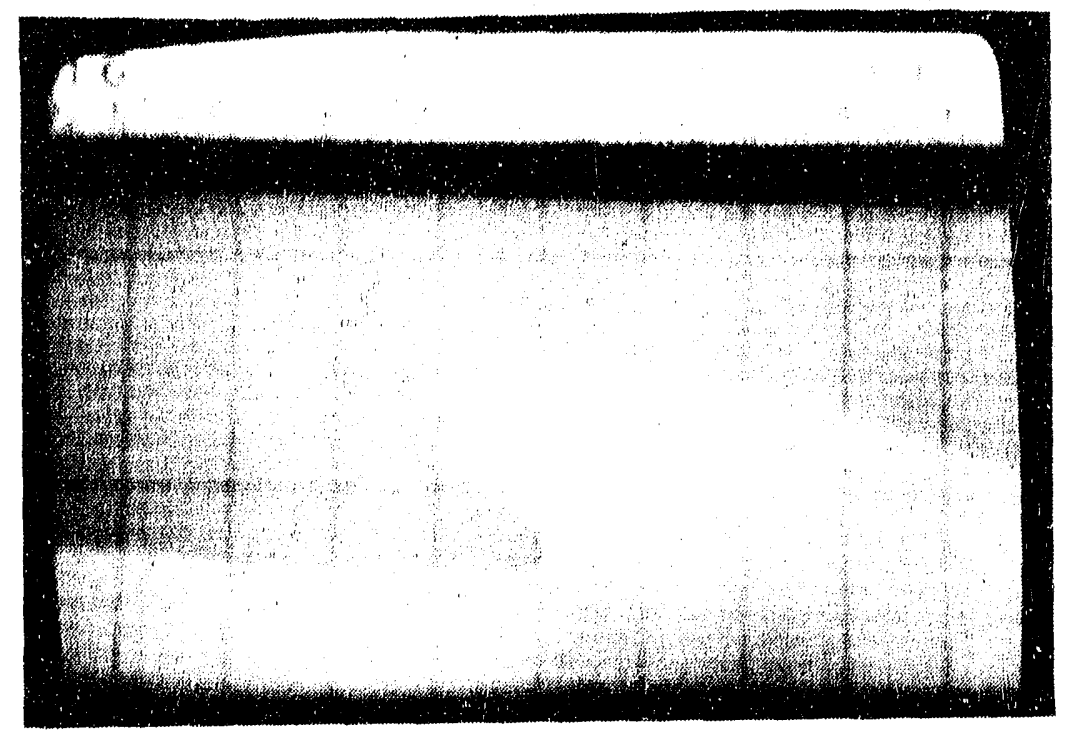

Figure 15: A Typical breaking wave

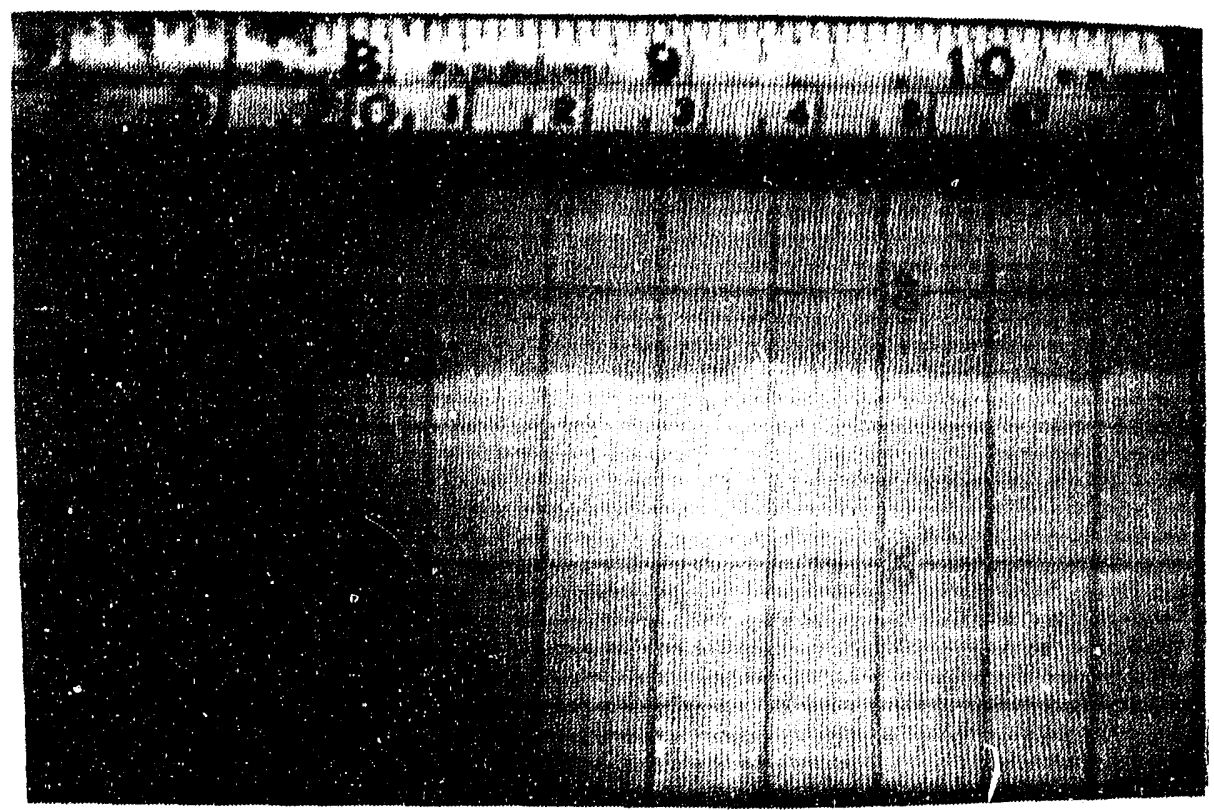

Figure 16: A wiggly interface behind the soliton 

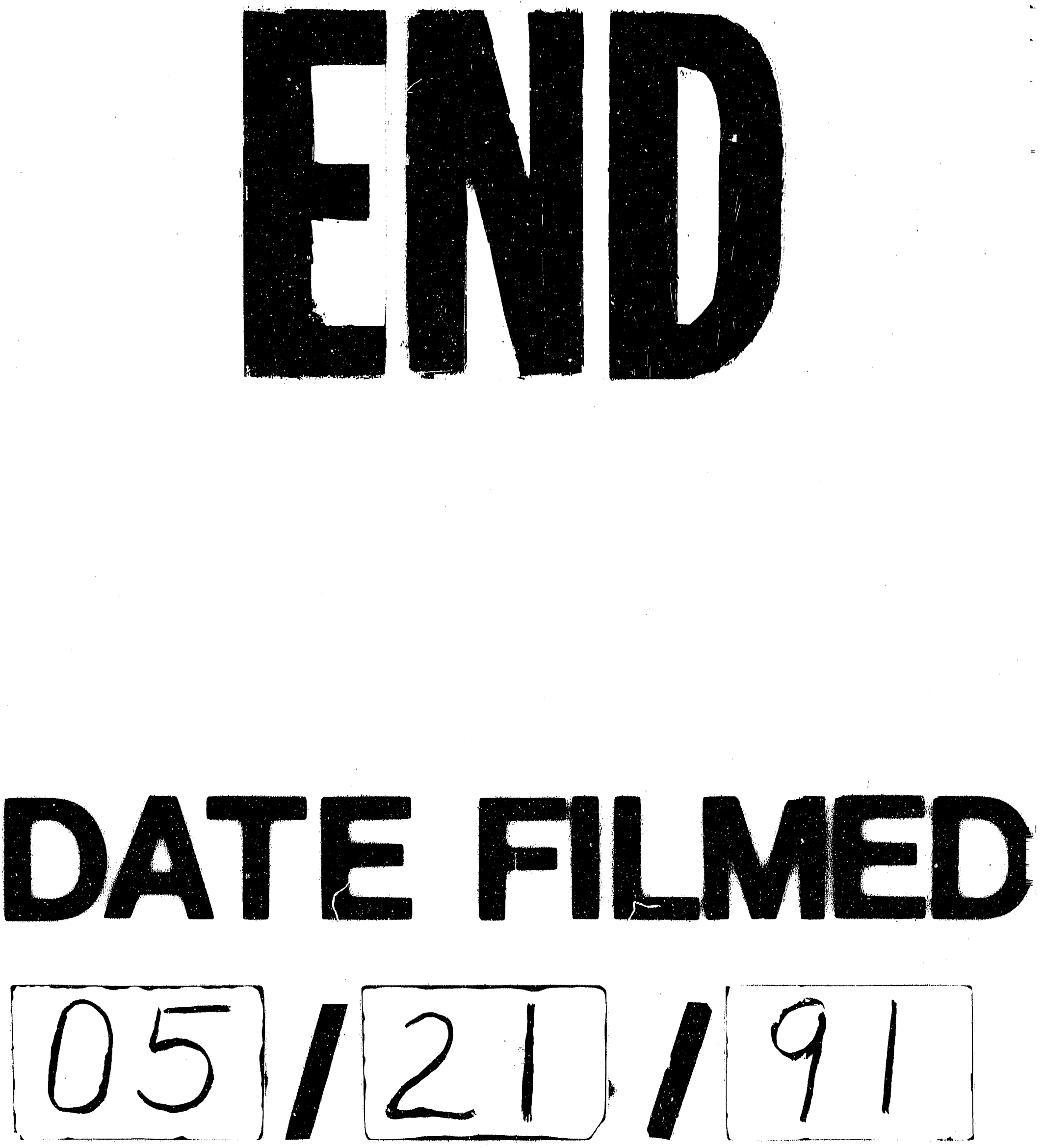
